\title{
Propiedades psicométricas de la versión piloto del Test de Predisposición hacia el Bullying (TPB) para niños entre 7 y 9 años de edad
}

Rosana Choy

Fabiola Henostroza

Vicente Rodríguez

Universidad de Lima

Recibido: 30 de abril del 2016 / Aprobado: 28 de junio del 2016

El estudio tuvo por objetivo analizar las propiedades psicométricas de la versión piloto del Test de Predisposición hacia el Bullying (TPB) trabajando con una muestra de niños entre 7 y 9 años de edad. Se utilizó un diseño de investigación transversal descriptivo, en el que se elaboró un instrumento evaluativo gráfico; se inició con la operacionalización de la variable, luego el diseño de una matriz de identificación de factores e indicadores de la variable, la estructuración de un instrumento piloto para niños de las edades seleccionadas, y se realizó una valoración de la representatividad de sus items por expertos. Finalmente, se aplicó la prueba, y se aportaron evidencias de validez y confiabilidad de las puntuaciones derivadas del instrumento.

bullying / niños / propiedades psicométricas / evidencias de validez

\section{Psychometric Properties in a Pilot Predisposition to Bullying Test (PBT) for Children Aged 7 to 9 Years}

The purpose of this study is to determine the psychometric properties of a pilot Predisposition to Bullying Test (PBT) in children aged between 7 and 9 years. Utilizing a cross-sectional method, we developed a graphic evaluation instrument which was applied to 91 intentionally selected children. The instrument fit approached the level conventionally recognized as adequate. The results show that the instrument has adequate validity and reliability.

\section{children bullying / psychometric properties / validity}

Correo electrónico: Rchoy@ulima.edu.pe 


\section{INTRODUCCIÓN}

La organización de la ONU para la Educación, la Ciencia y la Cultura (Unesco), a través de la Revista CEPAL, que analiza la realidad latinoamericana (n. ${ }^{\circ}$ 104, agosto 2011), proporciona datos referidos a la relación existente entre la violencia en estudiantes y el desempeño escolar. En el estudio llevado a cabo con 91223 estudiantes de sexto de primaria de 16 países latinoamericanos (Argentina, Brasil, Colombia, Costa Rica, Cuba, Chile, Ecuador, El Salvador, Guatemala, México, Nicaragua, Panamá, Paraguay, Perú, República Dominicana y Uruguay), se identificó que más del $50 \%$ de los estudiantes evaluados indicó haber sido víctima de alguna de las versiones de bullying por parte de sus compañeros de escuela. Según estos hallazgos, los alumnos víctimas de bullying logran un desempeño en lectura y matemáticas significativamente inferior al de quienes no sufren este tipo de maltrato.

Actualmente, en el Perú, funcionan 94746 centros educativos, de los cuales 71000 son públicos y se encontrarían sujetos a la llamada ley antibullying, Ley 29719 de Convivencia sin Violencia en las Instituciones Educativas; no obstante, para llevar a cabo acciones reales frente a esta problemática no solo educativa, sino también social, es necesario contar con instrumentos precisos que permitan identificar e intervenir sobre actitudes y conductas que se sustentan en violencia en el interior de los centros escolares.

En un estudio exploratorio, constituido por una muestra de 1703 estudiantes de $3 .^{\circ}$,
$4 .^{\circ}$ y $5 .^{\circ}$ de secundaria de escuelas públicas de Lima Metropolitana, se evaluó la prevalencia del cyberbullying utilizando las variables de género, grado escolar, rendimiento académico y las modalidades más utilizadas para la intimidación escolar. Se utilizó el Cuestionario Cyberbullying (Ortega, Calmaestra, y Mora Merchán, 2007), adaptado por el grupo de investigación. Los principales resultados indican que la prevalencia del cyberbullying es mayor por internet que por celular y existe una preponderancia de victimización hacia la mujer (García et al., 2010a).

En un estudio transversal llevado a cabo en colegios nacionales de Ayacucho, Cusco, Junín y Lima Este, se evaluó a 916 escolares utilizando el cuestionario validado de Oliveros y Barrientos (2007) con la finalidad de determinar la incidencia del bullying en colegios estatales de $5 .^{\circ} \mathrm{y}$ $6 .^{\circ}$ de primaria. Se encontró que la agresión más frecuente fue la verbal, en primer lugar; la física, en segundo lugar, y la discriminación, por último. Entre los tipos de agresiones verbales, se encuentra el colocar apodos, y entre los tipos de agresiones físicas, aparece el golpear y despojar dinero, así como escupir y forzar a cometer algún comportamiento. Asimismo, se encuentran otros hechos que alcanzan al $10 \%$ de la población encuestada, tales como realizar amenazas con armas blancas y de fuego (Oliveros et al., 2008).

Por lo anteriormente descrito y las problemáticas sustentadas en los diferentes hallazgos, surge la iniciativa de elaborar una prueba que permita identificar la predisposición del niño hacia el 
bullying y que cuente con las características psicométricas que faciliten ofrecer un instrumento de medición eficaz.

\section{LAS ACTITUDES}

Las actitudes han sido definidas como variables intermedias entre el estímulo, que es el objeto de la actitud, y la respuesta, que es la manifestación externa y medible. Están referidas hacia un objeto específico; es por eso que su capacidad de ser evaluadas es dirigida hacia la evaluación de algo en concreto. Su estructura se concibe sobre tres componentes: cognitivo, afectivo y conductual. La confección de escalas que midan las actitudes se ha basado en la designación de dichos componentes, puesto que la medición de las actitudes se basa en la evaluación de sus manifestaciones, las cuales son las reacciones valorativas ante creencias (dominio cognitivo), sentimientos (dominio afectivo) y comportamientos (dominio conductual) (Morales, 2006).

La mayoría de autores coincide en que la actitud es un constructo de la psicología social que es estable en el tiempo (Gawronski, 2007). Asimismo, el cambio de actitudes se manifiesta mediante la suplantación de la actitud nueva sobre la antigua, pero sin que esta desaparezca. Las personas pueden mantener varias actitudes en un mismo contexto, una actitud implícita y otra explícita (Ajzen, 2001).

Acerca de la relación entre las actitudes y los afectos, la preferencia es reservar el término afecto para las emociones generales (alegría y tristeza) y las emociones específicas (miedo, ira, envidia), estados que contienen valencias y activación (Ajzen, 2001).

Cualquier expresión de actitud es dependiente del contexto, pero se mantiene la idea de que las influencias contextuales producen variaciones alrededor de cierto valor que corresponde a la actitud. Los constructos de la actitud refieren que no hay necesidad de postular una entidad llamada actitud si los principios generales del juicio pueden contar como estabilidad, cambio, y pueden especificar las condiciones de la conducta de evaluación sin asumir disposiciones (Schwarz, 2007).

Se realizaron 3 experimentos en 41 participantes (24 mujeres y 17 varones) para evaluar la memoria y las actitudes. La finalidad fue investigar los mecanismos por debajo de la accesibilidad de los efectos de la información en medidas implícitas. Se evaluó cómo la información autogenerada afecta las medidas implícitas a través de la activación del conocimiento en la memoria asociativa o cómo las medidas implícitas son, en cambio, influenciadas por las experiencias subjetivas pertenecientes a la recuperación de información relevante de la memoria.

Los resultados indican que las medidas implícitas basadas en una respuesta compatible son influenciadas por la facilidad en la recuperación de información relevante de la memoria. En cambio, las medidas implícitas basadas en la compatibilidad del estímulo están influenciadas por el conocimiento directo, la activación en la memoria asociativa. Por tanto, los efectos del contexto en diferentes tipos de medidas implícitas pueden diferir con 
respecto a sus procesos subyacentes incluso cuando muestran efectos similares sobre un nivel superficial (Gawronski y Bodenhausen, 2005).

\section{BULLYING}

El bullying o acoso escolar está definido como una agresión de tipo sistemática cometida por uno o más miembros de un grupo, hacia otro(s) de forma seguida e intencional. Se expresa en una relación victimario-víctima, sin igualdad de poder (García et al., 2010b). Olerus (1979, como se citó en Oliveros et al., 2008) es considerado como uno de los precursores de los estudios longitudinales acerca del bullying, los cuales provienen de los países escandinavos y se originan treinta años atrás.

Todas las manifestaciones del $b u$ llying aparecen dentro de un contexto en donde el conocimiento y consentimiento de los actos de agresión es compartido. Las relaciones que se establecen son desequilibradas durante espacios de tiempo prolongados. El maltrato entre iguales ha sido enmarcado como conducta de acoso a partir del esquema dominio-sumisión (Avilés, 2011).

Es necesario realizar un análisis detallado de las acciones e interacciones dentro de la violencia escolar, ya que hechos esporádicos de agresión o delincuencia entre compañeros no se consideran como bullying; la variable acoso sistemático es una pieza fundamental para su conceptualización (Gómez, 2013).

\section{Enfoques que influyen en la aparición del bullying}

Los enfoques explicativos del origen de la violencia ofrecen una contextualización del escenario, importante de tomar en cuenta para el análisis del acoso escolar. Se tomarán en cuenta dos tipos de enfoque: el ecológico y el evolutivo (Díaz-Aguado, 2006).

Según el enfoque ecológico, el microsistema escolar está compuesto por el papel de los observadores, quienes representan un rol pasivo dentro de los actos de violencia, y son principales actores en el reforzamiento del victimario y en el aislamiento de la víctima. El microsistema también está compuesto por el profesorado, que constituye un rol influyente, puesto que es el protagonista de la falta de respuesta frente al problema de acoso escolar. Los profesores suelen atribuir las causas del problema a factores personales en los actores, y dejan de lado su responsabilidad en los actos de violencia. Frecuentemente, los profesores no forman parte de la solución, debido a su ausencia, ignorancia o falta de recursos para impedir la violencia. Esto está relacionado con el tratamiento inadecuado de la diversidad (Díaz-Aguado, 2006).

Por otro lado, el microsistema familiar indica que los principales antecedentes de un perfil agresivo en la escuela están compuestos por la ausencia de una relación afectiva y cálida dentro de la familia, la escasa disponibilidad para atender al niño, la ausencia de límites, la permisividad ante la violencia, los métodos 
coercitivos autoritarios y el castigo físico (Díaz-Aguado, 2006).

Desde el enfoque evolutivo, la explicación proviene de tomar en cuenta cualidades individuales, para lo cual convendría detectar qué habilidades no han sido desarrolladas adecuadamente. Existen tres tareas en la adaptación escolar: el establecimiento de vínculos, la estructuración de la conducta hacia los propios objetivos y la capacidad de cooperación (Díaz-Aguado, 2006).

\section{FACTORES DE RIESGO}

Es importante considerar la influencia de ciertos factores de riesgo existentes tanto en la víctima como en el acosador. En primer lugar, los factores que ubican al alumno en una situación de ser victimizado son los siguientes: la sumisión, es decir, la dependencia de otros niños y la identificación con el estereotipo femenino tradicional; el sexismo, en el cual existe la creencia de que el sometimiento y el uso de la violencia es inherente a la relación amical; la baja autoestima y autoeficacia, como una autopercepción y un autoconcepto sumamente negativos; los estilos de personalidad, referentes al miedo y la inseguridad emocional; la ansiedad; las escasas habilidades sociales, evidenciadas en la carencia de amigos y en las dificultades para establecer conexiones con grupos de apoyo social; el aislamiento; los rasgos físicos o culturales diferentes a la mayoría de niños del centro educativo; o pertenecer a una minoría, tener discapacidad o haber desarrollado trastornos psicopatológicos (Mendoza, 2012; Carozzo et al., 2012).
En segundo lugar, los factores que ubican al niño en una situación de victimario son la carencia de empatía, es decir, el desinterés por los sentimientos de sus compañeros; pérdida del control de impulsos, es decir, incapacidad para lidiar con emociones como el enojo; el bajo rendimiento escolar, evidenciado en una carencia de disponibilidad para hacer las tareas escolares; consumo de sustancias psicoactivas; la presencia de trastornos psicopatológicos, como trastornos de conducta, trastorno de control de impulsos, déficit de atención, trastorno negativista desafiante, trastorno disocial y trastorno adaptativo; identificación con un estereotipo masculino tradicional, caracterizado por actitudes como resolver los problemas a través del uso de la fuerza y el excesivo control; presencia de distorsiones cognitivas y manejo de una red social más amplia en comparación con las víctimas (Mendoza, 2012; Carozzo et al., 2012).

Existen otros factores importantes dentro del acoso escolar tales como los factores familiares, compuestos por la falta de comunicación dentro de la dinámica familiar, la sobreprotección, la ausencia de establecimiento de límites, la exposición crónica a la violencia y el uso de métodos disciplinarios autoritarios. Asimismo, existen los factores de influencia del centro educativo, como la existencia de un clima agresivo, las actitudes de indiferencia, la gestión disciplinaria verticalista, el hacinamiento provocado por una infraestructura no planificada y el burnout en docentes. Los medios de comunicación también cumplen un rol importante, puesto que el 
incremento de violencia en los programas de acceso libre permite que tanto víctimas como victimarios sean expuestos a episodios de agresión y estilos de conducta altamente impulsivos, además de escenas que infunden miedo e inseguridad. Por último, se pueden mencionar factores contextuales y ambientales como la baja calidad de vida, el estrés, y el divorcio o los problemas entre los padres (Carozzo et al., 2012).

\section{ANTECEDENTES}

El acoso escolar ha sido relacionado con diversas variables tales como la ansiedad social (Caballo et al., 2011), el ajuste familiar (Valdés, Martínez, y Torres, 2012), trastorno de estrés post traumático (Thormod, Dyregrov, y Cosmovici, 2012), rendimiento académico (Torregrosa et al., 2012), la ideación suicida (Bannink, Broeren, Van de Looij, De Waart, y Raat, 2014) y trastornos psicopatológicos (Felipe-Castaño, León-del-Barco, y Fajardo, 2013).

Mestre, Frías, y Samper (2004) relacionaron la empatía y la inadaptación escolar, junto con las variables género y grado escolar, en un estudio descriptivo comparativo, en el que se evaluó a 820 escolares de instituciones públicas con la versión española del Índice de Reactividad Interpersonal (Mestre, Frías, y Samper, 2004), la adaptación del Cuestionario de Intimidación y Maltrato entre Iguales Insebull (Avilés y Elices, 2007) y una encuesta de autodenominación sobre la posición ocupada. Se observó que sí existen diferencias significativas en todas las escalas de empatía según la variable género, y fueron las mujeres quienes puntuaron más alto en todas las escalas de empatía. No se encontraron diferencias significativas en inadaptación escolar en función de género y grado, pero sí en posición ocupada, y fueron las víctimas quienes presentaron los niveles más altos de inadaptación (García et al., 2010b).

La violencia ha sido identificada como medio de representaciones para los acosadores. Gómez (2013), en una investigación de enfoque cualitativo, realizó entrevistas y observaciones de campo con una muestra intencional, y encontró que una motivación importante para los intimidadores es conseguir un espacio representativo frente a sus compañeros; muchas veces es una forma de llamar la atención de los maestros, sobresalir socialmente y salir del anonimato buscando ser identificados y reconocidos. Para el acosador es difícil renunciar, puesto que debe evitar mostrar la pérdida de poder y fuerza. Asimismo, se identificó a los varones como principales promotores de violencia (Gómez, 2013).

Se evaluaron las atribuciones causales del acoso escolar en un estudio transversal que se llevó a cabo en 18 escuelas públicas de enseñanza media básica en Guadalajara, México. Los participantes fueron 1091 alumnos de secundaria entre los 12 y los 18 años de edad de ambos sexos, $62,5 \%$ mujeres y 75,5\% varones. Se aplicó un autoinforme a los alumnos y al personal escolar, es decir, directivos, intendentes y docentes. Los autoinformes fueron diseñados tomando como modelo los cuestionarios Intimidación y Maltrato 
entre Iguales en secundaria, de Ortega, Mora y Mora (1995); Evaluación de la Violencia en la Escuela y en el Ocio, de Díaz, Martínez y Seoane (2004), y Convivencia en los Centros Educativos, de Rodríguez (2005). Los resultados indican que tanto para los alumnos como para el personal de la escuela unas de las atribuciones causales son las conductas de broma con la finalidad de divertirse y supuestas situaciones conflictivas que provocan el acoso (Valadez, Gonzáles, Orozco, y Montes, 2011).

\section{Objetivos}

\section{General}

a) Elaborar un instrumento evaluativo gráfico para la identificación de actitudes hacia el bullying en niños de 7 a 9 años de edad

\section{Específicos}

a) Obtener evidencias de validez vinculadas a la estructura interna de la versión piloto del TPB

b) Estimar la confiabilidad de las puntuaciones derivadas de aplicar la versión piloto del TPB en una muestra de 91 niños de 7 a 9 años de edad

\section{Método}

\section{Participantes}

La versión piloto del instrumento se aplicó a 91 niñas y niños de 7 a 9 años de edad, de segundo, tercero y cuarto grado de primaria de un colegio público de Lima Metropolitana. Los niños y las niñas participantes fueron seleccionados de forma intencional y por oportunidad, debido a su disponibilidad y la posibilidad de tener acceso a ellos, considerando que se elige a los participantes de acuerdo al grado en que estos se ajustan a los criterios establecidos por el investigador (Rodríguez, Gil, y García, 1999).

Teniendo en cuenta que la aplicación del instrumento no implicaba ningún riesgo para los alumnos, la directora del plantel consideró que no era necesario solicitar el consentimiento informado a cada uno de los padres de los niños y niñas participantes en el estudio, y fue ella misma quien lo brindó.

\section{Procedimiento}

Tomando como referencia otros instrumentos utilizados para la medida de actitudes y conductas referidas al $b u$ llying, el equipo de trabajo del presente proyecto elaboró un instrumento acorde con la realidad de niñas y niños peruanos con edades comprendidas entre los 7 y 9 años. El instrumento de medida fue diseñado como un producto de la operacionalización de la variable a niveles observacionales mediante la definición de indicadores que reducían su nivel abstracto y hacían factible medirla. La variable se definió operacionalmente como situaciones de agresión física o verbal generadas por diferencias en las condiciones físicas como rasgos faciales, contextura física o discapacidad, y se midió a partir de la selección final que se consideró conveniente para cada una de las situaciones graficadas. 
Se construyó una matriz para descomponer en indicadores y obtener las posibilidades de elaboración de ítems en relación con dichas condiciones físicas. Luego, se procedió a elaborar el instrumento piloto, lo que hace referencia a la etapa inicial en torno a la creación de un modelo de la prueba. Al utilizar estímulos gráficos estructurados de manera no tradicional (tales como los característicos cuestionarios de lápiz y papel), se pretendió proporcionar al niño un material evaluativo con mayor atractivo, que permitiría recopilar información de forma más interactiva, congruente con sus capacidades cognitivas y visoperceptivas.

La versión piloto presentó 20 reactivos gráficos diseñados en proporción a los factores considerados, como las condiciones físicas y el género. Asimismo, el niño debía seleccionar, de tres alternativas gráficas, una respuesta que finalizara la historia de acuerdo con su conveniencia. Dichas respuestas fueron categorizadas como respuesta con conducta socialmente esperada, la cual valía cero puntos; respuesta con conducta de agresión verbal, valorada en un punto, y, finalmente, respuesta con conducta de agresión física, con una puntuación de dos.

Para poder solventar la adecuada representatividad de los reactivos, se solicitó la valoración de jueces u opinión informada de personas con autoridad en el tema y reconocidas por otros como expertos, que podían dar información, evidencia, juicios y valoraciones (Escobar-Pérez y CuervoMartínez, 2008). Así se reestructuró la prueba y se obtuvo un instrumento piloto de 10 ítems para emplear.
Cohen y Swerdlik (2006) plantean que la aplicación del instrumento piloto servirá para proporcionar los siguientes datos:

a) identificar ítems débiles o defectuosos, por ejemplo, ítems con significado ambiguo o indeterminado, distractores poco plausibles que no son elegidos por los sujetos, etc.;

b) determinar la dificultad de cada ítem, de tal modo que se pueda hacer una selección de acuerdo con la distribución de sus dificultades en función del uso previsto del test;

c) conocer, para cada ítem, su poder para discriminar entre sujetos altos y bajos en la variable que se está midiendo o entre diferentes grupos diagnósticos, cuando el uso previsto del test sea al diagnóstico clínico;

d) fijar cuántos ítems conformarán el test final;

e) establecer los límites de tiempo adecuados para el test resultante;

f) calcular las intercorrelaciones entre los ítems para examinar la homogeneidad del test.

\section{Resultados}

\section{Evidencias de validez vinculadas a la estructura interna}

En los Estándares para la Evaluación Educativa y Psicológica de 2010 (AERA, APA y NCME, 2014), se define la validez como una evaluación global del grado en el que la teoría y la evidencia apoyan las interpretaciones de las puntuaciones que se hacen en cada uso específico de los 
tests, y se ponen así de manifiesto la relevancia de la validez en el desarrollo y en la evaluación de los tests.

Por ello, se planteó analizar la estructura interna del instrumento a través de un análisis factorial exploratorio (Aiken, 2003). Para determinar si era posible realizar un análisis factorial, se verificó la medida de adecuación muestral KMO (Kaiser, Meyer y Olkin) y la prueba de esfericidad de Barlett (ver Tabla 1).

En la Tabla 2, observamos los autovalores extraídos para cada uno de los factores.
Se puede apreciar que tres de ellos son superiores a 1.00 y que, en conjunto, explican el $66,3 \%$ de la varianza. Luego de la extracción inicial y la aplicación de la extracción de varianza utilizando el método del eje principal, los tres primeros factores poseen una saturación cuadrada superior a 1.00 (Burga, 2006).

Del análisis factorial, podemos concluir que los factores quedan constituidos de la siguiente manera:

a) Factor 1 - Discapacidad: conformado por los ítems 8,4 y 5 , además de

Tabla 1

Medidas de adecuación de las variables al análisis factorial

\begin{tabular}{lll}
\hline Medida de adecuación muestral de Kaiser-Meyer-Olkin & .814 \\
Prueba de esfericidad de Bartlett & Chi-cuadrado & 351.268 \\
& aproximado & \\
& gl & 45 \\
& Sig. & .000 \\
\hline
\end{tabular}

Tabla 2

Varianza total explicada

\begin{tabular}{|c|c|c|c|c|c|c|}
\hline \multirow[t]{2}{*}{ Factor } & \multicolumn{3}{|c|}{ Autovalores iniciales } & \multicolumn{3}{|c|}{$\begin{array}{l}\text { Sumas de las saturaciones al cuadrado de la } \\
\text { extracción }\end{array}$} \\
\hline & Total & $\begin{array}{l}\% \text { de la } \\
\text { varianza }\end{array}$ & $\begin{array}{c}\% \\
\text { acumulado }\end{array}$ & Total & $\begin{array}{c}\% \text { de la } \\
\text { varianza }\end{array}$ & $\%$ acumulado \\
\hline 1 & 4.474 & 44.745 & 44.745 & 4.474 & 44.745 & 44.745 \\
\hline 2 & 1.145 & 11.447 & 56.191 & 1.145 & 11.447 & 56.191 \\
\hline 3 & 1.019 & 10.187 & 66.379 & 1.019 & 10.187 & 66.379 \\
\hline 4 & .803 & 8.031 & 74.410 & & & \\
\hline 5 & .711 & 7.108 & 81.518 & & & \\
\hline 6 & .496 & 4.960 & 86.478 & & & \\
\hline 7 & .415 & 4.150 & 90.628 & & & \\
\hline 8 & .411 & 4.106 & 94.734 & & & \\
\hline 9 & .318 & 3.180 & 97.914 & & & \\
\hline 10 & .209 & 2.086 & 100.000 & & & \\
\hline
\end{tabular}


los ítems 6 y 2, asociados al factor Contextura Física.

b) Factor 2 - Rasgos Faciales: conformado por el ítem 1, además de los ítems 10 y 9, asociados al factor Discapacidad.

c) Factor 3 - Contextura Física: conformado por los ítems 3 y 7 , asociados al factor Discapacidad.

\section{Confiabilidad}

Una de las primeras características que se debe determinar de una prueba recién elaborada es conocer si las puntuaciones derivadas de aplicar un test en una muestra específica resultan lo suficientemente confiables para medir lo que fue diseñada para medir (Aiken, 2003).

Para determinar la confiabilidad del TPB, se utilizó el coeficiente alfa de Cronbach, orientado a estimar la consistencia interna del instrumento con el cual se obtuvo resultados favorables.

El alfa de Cronbach es un coeficiente que permite estimar la fiabilidad de un instrumento de medida a través de un conjunto de ítems que se espera midan el mismo constructo o dimensión teórica. Cuanto más cerca se encuentre el valor del alfa a 1, mayor será la consistencia interna de los ítems analizados.

George y Mallery (2003, p. 231) sugieren los siguientes criterios para interpretar los coeficientes del alfa de Cronbach:

- Coeficiente alfa $>.9$ excelente

- Coeficiente alfa $>.8$ bueno

- Coeficiente alfa $>.7$ aceptable

- Coeficiente alfa >.6 cuestionable

- Coeficiente alfa $>.5$ pobre

- Coeficiente alfa $<.5$ inaceptable

Tomando en cuenta los criterios señalados líneas arriba, se procedió a estimar el valor del coeficiente total y de cada uno de los factores que componen la prueba, los cuales se presentan en la Tabla 3, en la cual observamos que los coeficientes del Factor 1, Factor 2 y Factor 3 se encuentran por encima de .70, lo cual indica que cuentan con un nivel aceptable. También se puede apreciar que el valor del coeficiente total se ubica por encima de 0.8 , con lo cual se demuestra que las puntuaciones derivadas del instrumento cuentan con buen nivel de confiabilidad.

\section{Tabla 3}

Alfa de Cronbach

\begin{tabular}{ccc}
\hline Variable & Alfa de Cronbach & Número de ítems \\
\hline Factor 1 & 0.773 & 5 \\
Factor 2 & 0.714 & 3 \\
Factor 3 & 0.752 & 2 \\
Test total & 0.858 & 10 \\
\hline
\end{tabular}




\section{CONCLUSIONES}

1. La versión piloto del Test de Predisposición hacia el Bullying (TPB) ha demostrado confiabilidad en la medición; el coeficiente alfa de Cronbach total se situó por encima de 0.8 .

2. Como aporte a las evidencias de validez referidas a la estructura interna, los resultados de la medida de adecuación muestral de Kaiser-Meyer-Olkin y la prueba de esfericidad Bartlett permiten realizar la aplicación del análisis factorial exploratorio.

3. La versión piloto del Test de Predisposición hacia el Bullying (TPB) es un instrumento multidimensional, pues se obtuvieron tres factores: Discapacidad, Rasgos Faciales y Contextura física.

4. Los factores que explican la variabilidad del desempeño de la prueba son Discapacidad, Rasgos Faciales y Contextura Física.

5. Las puntuaciones derivadas de aplicar el Test de Predisposición hacia el Bullying (TPB) en un grupo de niños y niñas de 7 a 9 años de edad son confiables.

\section{RefERENCIAS}

Aiken, L. R. (2003). Test psicológicos y evaluación. México: Pearson.

Ajzen, I. (2001). Nature and Operation of Attitudes. Annual Review of Psychology, 52, 27-58.
American Educational Research Association, American Psychological Association, y National Council on Measurement in Education. (2014). Standards for Educational and Psychological Testing. Washington D.C.: American Educational Research Association.

Avilés, J., Irutia, M., García-López, L. y Caballo, V. (2011). El maltrato entre iguales: "bullying". Behavioral Psychology, 19(1), 57-90.

Bannink, R., Broeren, S., Van de Looij, P., De Waart, y F. Raat, H. (2014). Plosone, 9(4). doi:10.1371/journal. pone.0094026

Burga, A. (2006). La unidimensionalidad en un instrumento de medición: perspectiva factorial. Revista de Psicología, 24(1), 53-80. Recuperado de http://www.redalyc.org/articulo. oa? id $=337829536003$

Caballo, V., Arias, B., Calderero, M., Salazar, I., y Irutia, J. (2011). Acoso escolar y ansiedad social en niños (I): análisis de su relación y desarrollo de nuevos instrumentos de evaluación. Behavioral Psychology, 19(3), 591-609.

Carozzo, J., Benites, L., Zapata L., y Horna V. (2012). El bullying no es un juego. Guía para todos. Lima: Observatorio sobre violencia y convivencia en la escuela.

Cohen, R. J., y Swerdlik, M. E. (2006). Pruebas y evaluación psicológicas. Introducción a las pruebas y a la medición. México, D. F.: McGraw-Hill. 
Díaz-Aguado, M. (2006). Del acoso escolar a la cooperación en las aulas. Madrid: Pearson.

Escobar-Pérez, J., y Cuervo-Martínez, A. (2008). Validez de contenido y juicio de expertos: una aproximación a su utilización. Avances en Medición, 6, 27-36. Recuperado de http://www. humanas.unal.edu.co/psicometria/files/7113/8574/5708/Articulo 3 juicio de expertos 27-36. pdf

Felipe-Castaño, E., León-del-Barco, B., y Fajardo, F. (2013). Perfiles psicopatológicos de los participantes en situaciones de acoso escolar en educación secundaria. Behavioral Psychology, 21(3), 475-490.

García, L., Orellana, O, Pomalaya, R., Yanac, E., Sotelo, L., Herrera, E., Sotelo, N., Chávez, H., García, N., Macazana, D., Orellana, D., y Fernandini, P. (2010a). Cyberbullying en escolares de educación secundaria de Lima Metropolitana. Revista de Investigación en Psicología Ilpsi, 13(2), 83-99.

García, L., Orellana, O, Pomalaya, R., Yanac, E., Sotelo, L., Herrera, E., Sotelo, N., Chavez, H., García, N., Macazana, D., Orellana, D., y Fernandini, P. (2010b). Intimidación entre iguales (bullying): empatía e inadaptación social en participantes del bullying. Revista de Investigación en Psicología Ilpsi, 14(2), 271-276.

Gawronski, B. (2007). Editorial: Attitudes Can be Measured! But What is an Attitude? Social Cognition, 25(5), 573-581.
Gawronski, B., y Bodenhausen, G. V. (2005). Accessibility effects on implicit social cognition: The role of knowledge activation and retrieval experiences. Journal of Personality and Social Psychology, 89, 672-685.

George, D., y Mallery, G. (2003). SPSS for Windows Step by Step: A simple Guide and Reference. (4..$^{\circ}$ ed.). Boston: Allyn y Bacon.

Gómez, A. (2013). Bullying: el poder de la violencia. Revista Mexicana de Investigación Educativa, 18(58), 839-870.

Gregory, R. J. (2001). Evaluación Psicológica. Historia, principios y aplicaciones. México, D. F.: Manual Moderno.

Gregory, R. J. (2012). Pruebas Psicológicas. Historia, principios y aplicaciones. México: Pearson.

Martínez, R. (2005). Psicometría: Teoría de los tests psicológicos y educativos. Madrid: Síntesis.

Mendoza, B. (2012). Bullying. Los múltiples rostros del abuso escolar. Córdova: Brujas.

Mestre, V., Frías, D., y Samper, P (2004). La medida de la empatía: análisis del Interpersonal Reactivity Index. Psicothema, 16(2), 255-260

Morales, P. (2006). Medición de actitudes en psicología y educación: construcción de escalas y problemas metodológicos. Madrid: Edisoter.

Oliveros, M., Figueroa, L., Mayorga, G., Cano, B., Quispe, Y., y Barrientos, A. (2008). Violencia escolar (bullying) en colegios estatales de primaria en el 
Perú. Revista Peruana de Pediatría, 61(4), 215-220.

Rodríguez, G., Gil, J., y García, E. (1999). Metodología de la investigación cualitativa. Málaga: Aljibe.

Schwarz, N. (2007). Attitude Construction: Evaluation in Context. Social Cognition, 25(5), 638-656.

Thormod, I., Dyregrov, A., y Cosmovici, E. (2012). Bullying and PTSD Symptoms. Abnormal Child Psychology, 40, 901-911.

Torregrosa, M., Inglés, C., García-Fernández, J., Gázquez, J., Díaz-Herrero, A., y
Bermejo, R. (2012). Conducta agresiva entre iguales y rendimiento académico en adolescentes españoles. Behavioral Psychology, 20(2), 263-280.

Valadez, I., Gonzáles, N., Orozco, M., y Montes, R. (2011). Atribuciones causales del maltrato entre iguales. Revista Mexicana de Investigación Educativa, 16(51), 1111-1136.

Valdés, A., Martínez, E., y Torres, G. (2012). Diferencias en la situación socioeconómica, clima y ajuste familiar de estudiantes con reportes de bu1lying y sin ellos. Psicología desde el Caribe, 29(3), 616-631. 
